\title{
Predicting Quality of Life and Mental Health During the First Year After Trauma Using Radial Basis Function Neural Network
}

\section{Zahra Sehat ( $\sim$ sehat.zahra426@gmail.com)}

Kashan University of Medical Sciences https://orcid.org/0000-0002-1712-7580

\section{Esmaeil Fakharian}

Kashan University of Medical Sciences

\section{Mojtaba Sehat}

Kashan University of Medical Sciences

\section{Abdollah Omidi}

Kashan University of Medical Sciences

\section{Research}

Keywords: Epidemiology, mental health, prediction, radial basis function neural network, quality of life, trauma

Posted Date: August 28th, 2020

DOI: https://doi.org/10.21203/rs.3.rs-62812/v1

License: (c) (i) This work is licensed under a Creative Commons Attribution 4.0 International License. Read Full License 


\title{
Predicting Quality Of Life And Mental Health During The First Year After Trauma Using Radial Basis Function Neural Network
}

\begin{abstract}
Background: This study aimed to investigate the quality of life (QOL) and mental health $(\mathrm{MH})$ post-trauma in the population over 15 years.
\end{abstract}

Methods: A cross-sectional population-based study, the cluster sampling method was used and 3880 people were interviewed randomly selected individuals between over 15 years in each household in the city of Kashan. Data were analyzed using chi-square, t-test. After data collection, a radial basis function neural network (RBFNN) architecture is exploited to predict the QOL category and MH status after traumatic injuries.

Results :The rate of trauma was $70.64(62.60-78.70)$ in 1000 annually, and $77.73 \%$ were male. $38.3 \%$ of people with trauma have suspected of having mental disorder and $53.3 \%$ of people with injury were in good condition of QOL. The risk of suspected MH disorders in people with trauma during the last year was 1.2(0.96-1.61), and the risk of bad QOL was 2.6(1.8-3.7). The obtained results reveal that the RBFNN model can predict the QOL category and MH status with a high level of accuracy (maximum $1 \%$ predicting error).

Conclusion :This study reveals several parameters associated with the MH and QOL after trauma. This parameter can be used in prediction outcomes and used to evaluate the care of people with injury. It can also be concluded that the RBFNN predictor can be used for predicting the QOL category and MH status with reasonable authenticity, efficiency, and accuracy.

Keywords: Epidemiology, mental health, prediction, radial basis function neural network, quality of life, trauma. 
Worldwide mortality rates after trauma significantly decreased and more injured patients survived (Celso et al., 2006; Twijnstra et al., 2010). Thus, non-fatal outcomes become more in demand (Moore et al., 2013). Assessment of QOL is essential to evaluate the success of treatment and to identify areas in which improvement is required 0(Ardolino et al., 2012).

$\mathrm{MH}$ in the World Health Organization was a combination of two dimensions: Negative and positive aspects. Negative of $\mathrm{MH}$ that indicates mental disorders, symptoms, and problems, the positive dimension of $\mathrm{MH}$ that includes positive emotions and personality traits such as selfesteem, overcoming the environment, a sense of integrity, and self-efficacy 0(Sasi e et al., 2004). QOL is an important part of a sense of $\mathrm{MH}$ and encompasses a dynamic and multidimensional concept: psychological and social (Khosrozadeh et al., 2015). The World Health Organization defines the QOL for each individual's understanding of their position in the context of cultural and value systems, and it emphasizes goals, expectations, standards, and interests. In this definition, QOL is a pervasive concept from physical health, Personal growth, Psychological states, the level of independence, Social relations, and Relationships with leading institutions of the environment are affected, and it depends on one's perception0 (Zheng et al., 2014; Levasseur et al., 2009). In other statements, QOL reflects how patients view their health state and have gained recognition as an evaluation criterion for medical treatments (Bowling et al., 2002; Gabbe et al., 2010; Williams et al., 2007).

General health in previous studies has shown to be a predictor of QOL in ICU patients, depression in trauma ICU patients (Toien et al., 2011). There were any previous studies that have investigated the impact of $\mathrm{MH}$ on Post trauma stress and QOL in trauma patients. There is a bright need, what were the most important demographic and injury characteristics, and the 
outcome variable (Disability, PTSD) could affect QOL and MH post-traumatic injuries. To fill in these gaps in knowledge, we performed a study with the following aim.

This study aimed to investigate the QOL and $\mathrm{MH}$ post-trauma in the population over 15 years and compared with the general population of Kashan. In this paper, the strong nonlinear mapping feature of radial basis function (RBF) neural networks is used to predict the QOL category and MH status of the post-traumatic injuries. The predicting results are close to the real values reported in the questionnaires.

\section{Method}

\section{Participants}

In a cross-sectional design used to examine factors influencing QOL and $\mathrm{MH}$ among traumatic people over 15 years, households residing in Kashan during 2018-9 were studied, the cluster sampling method was used.

\section{Procedure}

The city of Kashan was divided into five districts, according to the municipality. And blocks of each region are specified in the map. Thus, there were 32 blocks in each area, and the total number of blocks in the city was 160, and in each district of the block, 25 households were studied. Whole clusters of each region are numbered, and they were marked on the map, and blocks were selected. Randomly Simple and in every house between people over 15 years old were selected randomly for the interview, and in total, 3880 people were interviewed (Toien et al., 2011; Fakharian et al., 2019; Reihani et al., 2017; Rahimi-Movaghar et al., 2009; HafeziNejad et al., 2013) 
Demographic trauma information, mental health, and QOL were completed for all randomly selected individuals by the interviewer. In this study, trauma is any intentional or unintentional physical injury that following traumatic events have occurred, and this injury is needed for medical care.

According to the annual incidence of all injuries $(p)$ was assumed to be about 25 per 1000 person-years in 2013 (Fakharian et al., 2019), and the following formula was used to estimate the minimum needed sample size. Considering $d=1.5$

$$
n=\frac{z_{1-\alpha / 2}^{2} \times p \times q}{d^{2}}
$$

Due to the frequency of trauma that is $32.3 \%$, the required sample size for the study was multiplied by 1.5 in the design effect, and a total of 3875 study samples were determined (Khosrozadeh et al., 2015).

This study was approved by the ethics committee, and all patients have signed informed consent and assured for information confidentiality.

\section{Measures}

Trauma: In this study, the interviewers asked about any injury that had occurred during the past year, and the injury was any defined as any intentional or unintentional physical injury that needs medical service whether received or not and with or without intervention. Also, we categorized injury into minor ( $\leq 30$ days of disability) or major ( $>30$ days of disability), and two annual incidences were estimated from each mechanism of injury.

Mental Health: A 28-item General Health Questionnaire (GHQ-28) was used to assess mental health. GHQ-28 is a self-report questionnaire used to track persons with a mental disorder. This questionnaire focuses on two main categories of phenomena: One's inability to engage in a 'healthy and the emergence of new phenomena of a disabling nature. The 28 -item form of the 
Public Health Questionnaire has the advantage of being designed for all members of society. This questionnaire, as a screening tool, can determine the probability of a mental disorder in the individual. This questionnaire has four subscales: physical symptoms, anxiety and insomnia, Inadequate social action, and depression (Saadat et al., 2011; Tagharrobi et al., 2011).

In this study, the method of scoring the General Health Questionnaire is as follows: from points A to $\mathrm{D}$, scores of zero, one, two, and three. As a result, the scores on each of the subscales ranged from zero to 21 , and the total questionnaire ranged from zero to 84 . The scores of each subject were calculated separately on each scale and then subtracted for the four sub-scales, and the overall score was calculated. In this questionnaire, the lower score indicates better mental health. Based on the rating, the status of the individual in each of the subscales and the whole questionnaire was determined.

Quality of Life: Quality of Life Questionnaire (SF-12 ${ }^{1}$ ) was used to measure the QOL at the time of the study. The questionnaire contains eight subscales and assesses the QOL in terms of overall understanding of a person's health, physical functioning, physical health, physical problems, physical pain, social functioning, vitality and vital energy, and mental health (Levasseur et al., 2009; Bowling et al., 20042; Khosrozadeh et al., 2015). A higher score on this questionnaire indicates a higher QOL. In this study, the score of 48-37 indicates good QOL; a score of 36- 25 indicates the average QOL, and the score of 34-12 indicates poor QOL.

Post-traumatic stress disorder Checklist (PCL): This questionnaire consists of 17 items that 5 of which relate to the signs and symptoms of an accident re-experience, seven items on emotional and avoidance symptoms and symptoms, and five items in this list are for signs and symptoms of severe arousal (SHARON GIL., 2015; Weathers et al., 1993) .

1- Short Form-12 Quality of Life 
Scoring Method This list follows the IV-DSM criteria as scoring and having at least one symptom in each group of traumatic trauma re-experiencing symptoms, Emotional fatigue, and severe avoidance and arousal, and the sum of these scores are used as the cut-off point in the population. In this study, a cut-off point of 50 is used.

Disability: In this study, post-traumatic disability has been measured with the WHODAS II disability questionnaire. This 12-item questionnaire, designed by the World Health Organization (ICF). The questionnaire examines six areas, including communication and understanding, mobility, mobility, self-care, adjustment, and living with others, tasks related to daily living, and community participation (Ustün et al., 2010; (Bredemeier et al., 2014) .

In this study, the score of $12-24$ is equivalent to a mild disability, $48-25$ is equal to moderate disability, and 49 is above severe disability.

\section{Data Analysis}

All Statistical data analysis was performed using SPSS 22 software. Chi-square and T-tests were used to examine the differences between the two variables. Logistic regression analysis was used to examine the variables that were significantly associated with trauma outcomes. The significance level was considered less than 0.05. Univariate analysis was used to investigate the relationship between variables and trauma outcomes.

\section{Radial Basis Function Neural Networks}

In this paper, the RBFNN-based algorithm of (Baghaee et al., 2012) is modified to be used to predict the QOL category and MH status of the post-traumatic injuries. The middle layer of RBFNN, named the hidden layer that consists of $\mathrm{H}$ basic nonlinear Gaussian activation functions to map their input vector to their output, vector a (Fig. 1). Input nodes directly pass the input 
values to the hidden neurons, and the first layer is not weighted. Outputs of the networks are produced multiplying this vector by the weights matrix $\mathrm{W}$, by a linear summation of the resulting matrix (Demuth et al., 2015; Chen et al., 1991; Leonard., 1991; Tarassenko et al., 1994) . As shown in the schematic diagram of RBFNN with $\mathrm{H}$ basic neuron in the hidden layer and scalar

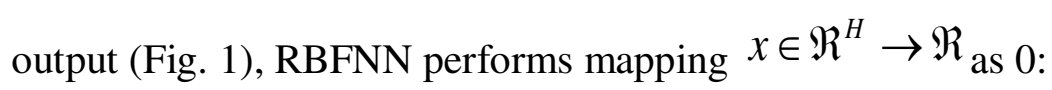

$$
f(x)=w_{0}+\sum_{k=1}^{H} w_{k} \phi\left(\left\|x_{k}-\mu_{k}\right\|\right)
$$

Where $x \in \mathfrak{R}^{H}$ is the input vector, $\phi($.$) is a given function from \mathfrak{R}^{+}$to $\mathfrak{R}$, and $w_{k}$ is the weighting factor of the parameter.

Fig. 1 Schematic of RBFNN 0( Chen et al., 1991) .

The hidden layer output is defined as:

$$
a_{h k}=e^{\left(-\frac{\left\|x_{k}-\mu_{h}\right\|^{2}}{\sigma_{h}^{2}}\right)}
$$

Each node has $N+1$ parameters. $\sigma_{h}$ determines what distance in the input space the $h^{\text {th }}$ unit will have a significant influence. Some other non-linear activation functions can be used as the basic function of the kernel in place of Gaussian function (Demuth et al., 2015). The network's output is given by:

$$
y_{m k}=\sum_{h=1}^{H} w_{m h} a_{h k}
$$

The training algorithm of the RBF network aims to find the optimal values for $N+1$ centre and widths of kernels, as well as output layer's weighting factors to minimize the following objective function to make $T_{k}$ as close as possible to $Y_{k}$ (Demuth et al., 2015; Chen et al., 1991) . 


$$
E(\mu, \sigma, w)=\sum_{k=1}^{K}\left\|Y_{k}-T_{k}\right\|^{2}
$$

Choosing the optimal kernel centres, among the exiting input patterns, back-propagation (BP), decoupled two-layer training, and hybrid method (Leonard., 1991) have been reported to optimize the above objective function based on orthogonal least square (OLS) algorithm (Chen et al., 1991) and exact fit (EF) method 0(Demuth et al., 2015). The goal of the EF training method is to find the I/O relationship between spaces with zero error, and so, the number of the hiddenlayer neurons is chosen to be as equal as the number of the input vector. Hence, it is enough to set the center of each kernel on a specific input vector (Demuth et al., 2015; Chen et al., 1991; Leonard., 1991). This idea makes the training procedure fast and simple, and RBF-ANN suitable for iterative computations. By providing a set of the input $x(k)$ and the corresponding desired/target output $T(k)$ values of the weights, $w_{k}$ can be determined using the linear OLS

method. Different activation functions can be defined. However, in this paper, the $\phi($.$) is selected$ as the Gaussian activation function of (3) to RBFNN to match the performance of the two-layer neural network closely. To use the OLS method, it is essential to consider RBFNN as a special case of a linear regression model:

$$
T(k)=\sum_{i=1}^{M} p_{i}(k) \theta_{i}+\varepsilon(k)
$$

$p_{i}(k)$ are known as repressors and are function of $x(k)$ namely $p_{i}(k)=p_{i}(x(k))$. Here, it is assumed that the error signal is uncorrelated to the repressors.

Matrix representation of OLS is given by arranging (4) as:

$$
T=P \Theta+E
$$


where

$$
\begin{aligned}
T & =\left[\begin{array}{ll}
T(1) \mathrm{K} & T(H)
\end{array}\right]^{T} \\
P & =\left[\begin{array}{ll}
p_{1}, \mathrm{~K} & p_{M}
\end{array}\right], p_{i}=\left[\begin{array}{ll}
p_{i}(1) \mathrm{K} & p_{i}(N)
\end{array}\right]^{T}, 1 \leq i \leq M \\
\Theta & =\left[\begin{array}{ll}
\theta_{1} \Lambda & \theta_{M}
\end{array}\right]^{T} \\
E & =\left[\begin{array}{ll}
\varepsilon(1) \mathrm{K} & \varepsilon(N)
\end{array}\right]^{T}
\end{aligned}
$$

A set of basis vectors is formed by regressor vectors $\boldsymbol{p}_{\boldsymbol{i}}$, and the OLS solution $\boldsymbol{\Theta}$ is obtained so that $\boldsymbol{P} \boldsymbol{\Theta}$ is the projection of $\boldsymbol{T}$ onto space spanned by these vectors. This means that the square of the projection $\boldsymbol{P} \boldsymbol{\Theta}$ is part of the desired output energy that can be counted by regressors. The OLS transforms the set of orthogonal vectors and provides the possibility to calculate the contribution from each basis vector to target output energy. The regression matrix can be decomposed as 0( Chen et al., 1991):

$$
P=W A
$$

where $\mathrm{A}$ is an $M \times M$ triangular matrix that is expressed as:

$$
A=\left[\begin{array}{ccccc}
1 & \alpha_{12} & \alpha_{13} & \Lambda & \alpha_{1 M} \\
0 & 1 & \alpha_{23} & \Lambda & \alpha_{2 M} \\
0 & 0 & \mathrm{O} & \mathrm{O} & \mathrm{M} \\
\mathrm{M} & \mathrm{O} & \mathrm{O} & 1 & \alpha_{M-1 M} \\
0 & \Lambda & 0 & 0 & 1
\end{array}\right]
$$

and $\boldsymbol{W}$ is an $N \times M$ matrix including columns $\boldsymbol{w}_{\boldsymbol{i}}$ so that $\boldsymbol{W}^{T} \boldsymbol{W}=\boldsymbol{H}$, where $\boldsymbol{H}$ is diagonal with elements $h_{i}$ :

$$
h_{i}=w_{i}^{T} w_{i}=\sum_{k=1}^{H} w_{i}(k) w_{i}(k) ; 1 \leq k \leq M
$$

Because space spanned by the set of orthogonal basis vectors $\boldsymbol{w}_{i}$ is same as ones spanned by set of $\boldsymbol{p}_{\boldsymbol{i}}$, we can write (5) as: 


$$
T=W g+E
$$

The solution $\boldsymbol{g}$ is given by:

$$
G=H^{-1} W^{T} T
$$

or

$$
g_{i}=w_{i}^{T} T /\left(w_{i}^{T} w_{i}\right)
$$

and $\boldsymbol{g}$ and $\boldsymbol{\Theta}$ satisfy the triangular system so that we have:

$$
A \Theta=g
$$

Based on classical Gram-Schmidt 0( Chen et al., 1991), method, the computation procedure for updating weighting factors are calculated as follows:

$$
\left.\begin{array}{l}
w_{1}=p_{1} \\
\alpha_{i k}=w_{i}^{T} p_{k} /\left(w_{i}^{T} w_{i}\right) \quad 1 \leq i \leq k \\
w_{k}=p_{k}-\sum_{i=1}^{k=1} \alpha_{i k} w_{i}
\end{array}\right\} k=2, \mathrm{~K}, M
$$

The error reduction ratio due to $\boldsymbol{w}_{i}$ is defined as 00(Chen et al., 1991):

$$
[\mathrm{err}]_{i}=g_{i} w_{i}^{T} w_{i} /\left(d^{T} d\right), \quad 1 \leq i \leq M
$$

At the end, the regressor selection procedure is summarized as 0( Chen et al., 1991):

As the first step, for $1 \leq i \leq M$, compute:

$$
\begin{aligned}
& w_{1}^{(i)}=p_{i} \\
& g_{1}^{(i)}=\left(w_{1}^{(i)}\right)^{T} d /\left(\left(w_{1}^{(i)}\right)^{T} w_{1}^{(i)}\right) \\
& {[\operatorname{err}]_{1}^{(i)}=\left(g_{1}^{(i)}\right)^{2}\left(w_{1}^{(i)}\right)^{T} w_{1}^{(i)} /\left(d^{T} d\right)}
\end{aligned}
$$


Find,

$$
[e r r]_{1}^{(i 1)}=\max \left\{[e r r]_{1}^{(i)}, 1 \leq i \leq M\right\}
$$

and select

$$
w_{1}=w_{1}^{(i 1)}=p_{i 1}
$$

At the kth step $(k \geq 2)$, for $1 \leq i \leq M, I \neq i_{1}, I \neq i_{k-1}$, compute

$$
\left.\begin{array}{c}
\alpha_{j k}^{(i)}=w_{j}^{T} p_{i} /\left(w_{j}^{T} w_{j}\right), 1 \leq j \leq k \\
w_{1}^{(i)}=p_{i}-\sum_{j=1}^{k-1} \alpha_{j k}^{(i)} w_{j} \\
g_{k}^{(i)}=\left(w_{k}^{(i)}\right)^{T} d /\left(\left(w_{k}^{(i)}\right)^{T} w_{k}^{(i)}\right) \\
{[e r r]_{k}^{(i)}=\left(g_{k}^{(i)}\right)^{2}\left(w_{k}^{(i)}\right)^{T} w_{k}^{(i)} /\left(d^{T} d\right)}
\end{array}\right\}
$$

Find

$$
[e r r]_{k}^{(i 1)}=\max \left\{[e r r]_{k}^{(i)}, 1 \leq i \leq M, i \neq i_{1}, \mathrm{~K}, i \neq i_{k-1}\right\}
$$

and select

$$
w_{k}=w_{k}^{(i k)}=p_{i k}-\sum_{j=1}^{k-1} \alpha_{j k} w_{j}
$$

where $\alpha_{j k}=\alpha_{j k}^{(i k)}, 1 \leq j<k$. The training procedure is terminated at $M^{\text {th }}$ step when the following condition is satisfied:

$1-\sum_{j=1}^{M}[e r r]_{j}<\varepsilon$

where $\varepsilon$ is predefined tolerance. Generally, training of RBF networks is some order of magnitude faster than the same size multi-layer perceptron (MLP) networks (Demuth et al., 2015). To 
compare RBFNN, and MLP-ANN, two parameters have defined that show the difference between output and target data of the system. Here, the correlation coefficient $(R)$ and root mean square error (RMSE) is used to evaluate the performance of both RBFNN and MLP-ANN. These parameters are defined as:

$$
\begin{gathered}
R=\frac{\sum_{i=1}^{N}\left(t_{i}-\bar{t}\right)\left(O_{i}-\bar{O}\right)}{\sqrt{\sum_{i=1}^{N}\left(t_{i}-\bar{t}\right)^{2}\left(O_{i}-\bar{O}\right)^{2}}} \\
R M S E=\sqrt{\frac{1}{N} \sum_{i=1}^{N}\left(t_{i}-O_{i}\right)^{2}}
\end{gathered}
$$

The more correlation coefficient shows more accurate regression. Also, fewer RMSE is a vestige for the high ability of method for estimation of data. The variations of mismatch versus iterations for the RBFNN predictor is shown in Fig. 2.

Fig. 2. Variations of mismatch versus iterations for the RBFNN predictor.

\section{Results}

\section{People with trauma}

In this study, the rate of trauma in one year per 100 people was 7.061 , and $77.73 \%$ of the patients with trauma were male. $161(58.7 \%)$ minor injuries (123 males; 38 female) and 113 (41.4\%) major injuries (90male;23females), also 50\% of trauma occurred among people aged 20 to 40 years. $75.7 \%$ of people with trauma were married. The most mechanism of injury (51\%) was related to traffic accidents, and among the traffic accidents, the highest cause was motorcycles $(70.71 \%)$.

People with trauma, 67\% had more than 24 hours' hospitalization. Among hospitalized patients, $41.40 \%$ received surgical treatment, and $58.39 \%$ received non-surgical treatment. 
In this study, it was found that $17.2 \%$ of people with traumatic injuries have post-traumatic stress disorder, and $89.3 \%$ have a mild disability, and $10.7 \%$ have moderate disabilities and no case that reported severe disabilities.

The computer program was performed on MATLAB (version 2018a, The Math Works Inc., USA) environment. These values are very satisfying. The prediction performance of this method shows the advantages of RBFNN. It is rapid, easy to operate, and not expensive. The findings demonstrate the learning and predicting the potential of the RBFNN model in financial applications. Furthermore, these results indicate that RBFNN can be a useful tool for prediction in return to work post-trauma.

\section{Mental Health and Quality of life compared with the general population}

In total, people that have been investigated, GHQ-28, result that $61.7 \%$ have normal results and $38.3 \%$ have suspected of having mental disorder results that this result in people with trauma have the same model and $33.6 \%$ of people with trauma had symptoms and $66.4 \%$ have a normal result. In anxiety and insomnia part, $30.3 \%$ of people with trauma had symptoms of the anxiety and insomnia. In function part, $13.6 \%$ of people with trauma were suspected of having disorders. In the depression part, $76.3 \%$ of people with trauma were suspected of having disorder depression. Table 1shows the comparison of $\mathrm{MH}$ and QOL in people with or without trauma. In this study, the QOL in most of the population (53.3\%) was in good condition, and those with trauma during one year ago, most (49\%) had a moderate QOL, whereas those without trauma (55\%) had a good QOL (Figs. 3-4).

Table 1- Compare mental health and QOL in people with or without trauma

Fig. 3 QOL in people with or without trauma.
Fig. 4 Mental health in people with or without trauma. 
In this study, among people with trauma during one year ago, most people were normal levels of $\mathrm{MH}(66.4 \%)$. There was a significant relationship between $\mathrm{MH}$ and gender $(P=.001)$, with trauma women and men having more suspected of having mental disorder mental health. Also, there was a significant relationship between $\mathrm{MH}$ among different age groups, so that trauma patients had suspected of having mental disorder $(P=0.05)$. This correlation was also found for characteristics such as marital status, insurance coverage, chronic diseases such as diabetes and hypertension, also the level of education. Among those with trauma in the last year and according to the SF-12 questionnaire QOL, most of the subjects had a moderate and good QOL (92.43\%). There was no significant relationship between $\mathrm{MH}$ and gender $(P=0.06)$. Also, there was a significant relationship between QOL in different age groups so that those with trauma had a higher average QOL $(P=0.00)$. This relationship was also seen for characteristics such as marital status, insurance coverage, chronic illnesses such as diabetes and hypertension, as well as education levels. Table 2 shows the Demographic characteristics based on $\mathrm{MH}$ and QOL status in people with trauma

Table 2-Demographic characteristics based on Mental Health and QOL status in people with trauma

In this study, there was a significant relationship between the mechanism of trauma and $\mathrm{MH}(P$ $=0.01)$. In all trauma mechanisms, the rate of suspected of having mental disorder $\mathrm{MH}$ was higher, but $\mathrm{MH}$ was not correlated with the number of injuries $(P=0.9)$. Also, $\mathrm{MH}$ was significantly associated with surgery to treat injuries $(P=0.05)$. In this study, there was a significant relationship between the mechanism of trauma and QOL $(P=0.08)$. The average QOL was higher for all trauma mechanisms QOL with the number of injuries caused $(P=0.04)$. 
There was also a significant relationship with surgery to treat injuries $(P=0.05)$. Table 3 shows the relationship between $\mathrm{MH}$ and QOL and trauma-related variables.

Table 3- Injury characteristics based on mental health and quality of life status

Also, there was a significant relationship between the level of the $\mathrm{MH}$ of people who have trauma and their QOL $(p=0.00)$. Most people with poor QOL also suspected of having mental disorder mental health (23.6\%). Table 4 shows the Correlation between General Health and QOL in people with trauma during one year ago.

Table 4- Correlation between General Health and QOL

Using the RBFNN, we obtain the predicted values of QOL and MH after trauma, and the result is presented in table 5. The result of RBFNN shows that Having a history of trauma can affect people's QOL. And there are factors such as male gender, being married, being over five years old, having low education, having a specific illness, and having no insurance. Also, this prediction method also showed that trauma has little effect on $\mathrm{MH}$.

\section{Table 5-the values of Mental health and QOL predicted with RBFNN}

\section{Discussion}

The main findings in this study of people with trauma, $77.73 \%$ of the people were male. The highest rate of injury occurred among people aged 20 to 40 years, $75.7 \%$ of them were married. The most mechanism of trauma was related to traffic accidents (51\%), and $70.71 \%$ of them were motorcycles.

This study provides parameters significantly associated with $\mathrm{MH}$ and QOL in the study with trauma. This parameter can be used in prediction outcomes and used to evaluate the care of people with trauma. Results of MH testing showed $33.6 \%$ of people with trauma had symptoms, 
and $30.3 \%$ of people with trauma had symptoms of anxiety and insomnia, and $13.6 \%$ of people with trauma was suspected of having function disorder, and $76.3 \%$ was suspected of having disorder depression. People with trauma, most (49\%) had a moderate QOL.

It might have been expected that there had been more improvement in other domains than physical functioning, pain, and social functioning. On the other hand, the physical and pain domains were those most impaired, compared to the general population. In truth, this item was studied, but for a reason many for expounding in one paper, this we will propose in other my papers.

Some studies showed poorer outcomes, e.g., in QOL and greater morbidity for older people with trauma (Toien et al., 2011; Pagulayan et al., 2006). Due to the complexity of trauma, especially TBI and the lengthy process of rehabilitation, the rate of recovery may vary depending on time after injury (Ustün et al., 2010; Bredemeier et al., 2014). The relationship between time after injury and QOL in people with TBI has been well documented in several studies (Pagulayan et al., 2006; Truelle et al., 2010) . But there are few studies about other trauma and General Health and QOL.

Multi previous studies have been identified that compared QOL in trauma patients with the general population or a healthy control group, all of these studies reported significantly lower scores for the patients compared with the general population/control group (Bowling et al., 2002; Toien et al., 2011).

In the study on the association of patient and trauma characteristics with the health-related QOL in a Dutch trauma population in 2017 (Gunning et al., 2017). A total of patients was included for analyses. Compared to the eligible population, included patients were significantly older, 
more severely injured, more often admitted in the ICU, and had a longer admission duration. The SF-12 and EQ-5D were significantly lower compared to the Dutch reference population. Gender and age and marital state and age group showed to have an influence on GH and QOL in this study. Previous studies showed that there was a difference between this parameter and GH and QAL (Williams et al., 2007; Toien et al., 2011; Gunning et al., 2017).

The study also identified that $\mathrm{MH}$ and QOL in people with trauma are lower than others. This can determine the importance of $\mathrm{MH}$ in individuals. To judge whether trauma has impaired the QOL and the MH of individuals or whether these conditions existed before the trauma, it is important to have information on the QOL and $\mathrm{MH}$ of individuals before the trauma. But Considering that, this study is population-based, the ability to measure, and even people. Before, the trauma is very difficult.

\section{Conclusion}

This study reveals several parameters associated with the GH and QOL after trauma. These factors were age, gender, marital status, education, illness, mechanism of trauma, treatment This parameter can be used in prediction outcomes and used to evaluate the care of people's trauma.

Trauma-related injuries have not made a significant difference in different $\mathrm{MH}$ issues But it does change people's QOL. This decrease in the QOL of traumatic individuals is more pronounced among people who are more severely disabled and have post-traumatic stress disorder. It can also be concluded that the RBFNN predictor can be used for predicting of the QOL and MH posttrauma with reasonable authenticity, efficiency, and accuracy. 


\section{Declarations}

\section{- Ethics approval and consent to participate}

This study was being approved by ethical committee of Kashan Medical science university, Kashan, Iran. Code of Ethics (1397,094).

Before starting the interview, for each of the participants was described objectives research. Also has been mentioned that their information would not be cited and their information will remain confidential to the researcher than If they agree to participate in the research, they were informed consent to participate in the research.

In this study, we tried to comply do all human rights permissions

\section{- Consent for publication}

For each of the participants was described objectives research. Also has been mentioned that their information would not be cited and their information will remain confidential to the researcher .

\section{- Availability of data and material}

not applicable

\section{- Competing interest}

Authors report no commercial or other association that might pose a conflict of interest with respect to this research. 


\section{- Funding}

This study was from a Ph.D. dissertation and supported by a grant from the Kashan Medical science university Foundation. The Research project code is 97148 , affiliated to Kashan University of Medical Sciences.

\section{- Authors contributions}

ZS, MS, EF, AO: designed the study, ZS, MS: managed data collection, ZS: analyzed data and composed manuscript draft and edited final manuscript performed the literature search and contributed to the manuscript draft. MS contributed to data collection, analysis, and drafting and also edited the draft. All four authors read and approved the final manuscript.

\section{- Acknowledgments}

We would like to thank the Trauma research Center of Kashan University of Medical Sciences and the students and faculty at the University for their support and assistance with this project.

\section{References}

Celso B, Tepas J, Langland-Orban B, Pracht E, Papa L, Lottenberg L, et al. A systematic review and meta-analysis comparing outcome of severely injured patients treated in trauma centers following the establishment of trauma systems. J Trauma. 2006;60:371 -8. discussion 378 .

Twijnstra MJ, Moons KG, Simmermacher RK, Leenen LP. Regional trauma system reduces mortality and changes admission rates: a before and after study. Ann Surg. 2010; 251:339-43. 
Moore L, Stelfox HT, Boutin A, Turgeon AF. Trauma center performance indicators for nonfatal outcomes: a scoping review of the literature. J Trauma Acute Care Surg. 2013; 74:1331-43.

Ardolino A, Sleat G, Willett K. Outcome measurements in major trauma- results of a consensus meeting. Injury. 2012;43(10):1662-6.

Sasi A. Willmott · Jed A. P. Boardman · Carol A. Henshaw · Peter W. Jones Understanding General Health Questionnaire (GHQ-28) score and its threshold. Soc Psychiatry Psychiatr Epidemiol .2004; 39 : 613-617

Khosrozadeh H, Alavi NM, Gilasi H, Izadi M. Oral health-related quality of life in older people in Kashan/Iran 2015.Nurs Midwifery Stud .2017;6:182-8.

Zheng QL, Tian Q, Hao C, Gu J, Lucas-Carrasco R, Tao JT, et al. The role of quality of care and attitude towards disability in the relationship between severity of disability and quality of life: fidings from a cross-sectional survey among people with physical disability in China. Health and Quality of Life Outcomes. 2014; 12(1):1-22.

Levasseur ML,St-Cyr Tribble D,Desrosiers J. Meaning of quality of life for older adults: Importance of human functioning components. Archives of gerontology and geriatrics. 2009; 49(2):91-100.

Bowling A, Banister D, Sutton S, Evans O, Windsor J. A multidimensional model of the quality of life in older age. Aging Mental Health. 2002; 6(4):355-71.

Gabbe BJ, Sutherland AM, Hart MJ, Cameron PA: Population-based capture of long-term functional and quality of life outcomes after major trauma: the experiences of the Victorian State Trauma Registry. J Trauma 2010, 69:532-536. 
L. Williams, David R. Williams, Dan J. Stein, Soraya Seedat, Pamela B. Jackson, and Hashim Mooma. Multiple Traumatic Events and Psychological Distress: The South Africa Stress and Health Study Stacey. J Trauma Stress. 2007 October; 20(5): 845-855.

Kirsti Tøien1, Inger S Bredal, Laila Skogstad, Hilde Myhren and Øivind Ekeberg.Health related quality of life in trauma patients.Data from a one-year follow up study compared with the general population. Scandinavian Journal of Trauma, Resuscitation and Emergency Medicine 2011:19(22) .

Fakharian E, Sehat Z, Sehat M. Traumatic spine injury in Kashan, Iran. Journal of Emergency Practice and Trauma 2019; 5(2): 65-70.

Reihani H, Pirazghandi H, Bolvardi E, Ebrahimi M, Pishbin E, Ahmadi K, Safdarian M, Saadat S , Rahimi-Movaghar V. Assessment of mechanism, type and severity of injury in multiple trauma patients: A cross sectional study of a trauma center in Iran .Chinese Journal of Traumatology.2017; 20: 75-80.

Rahimi-Movaghar V, Zarei MR, Saadat S, Rasouli MR, Nouri M. Road traffic crashes in Iran from 1997 to 2007. Int J Inj Contr Saf Promot. 2009;16:179-81

Hafezi-Nejad N, Rahimi-Movaghar A, Motevalian A, Amin-Esmaeili M, Sharifi V, Hajebi A, Radgoodarzi R, Hefazi M, Eslami V, Saadat S, Rahimi-Movaghar V .A nationwide population-based study on incidence and cost of non-fatal injuries in Iran. Inj Prev 2014; 0:1 5. https://doi.org/10.1136/injuryprev-2013-041127

Soheil Saadat, Mostafa Mafi and Mahdi Sharif-Alhoseini Population based estimates of nonfatal injuries in the capital of Iran. BMC Public Health 2011, 11:608.1471-2458/11/608. 
Tagharrobi Z, Sharif K, Sooky Z. [Psychometric evaluation of Shah version of modifid Barthel index in elderly people residing in Kashan Golabchi nursing home (Persian)]. Journal of Kashan University of Medical Sciences. 2011; 15(3):213-24.

Levasseur ML,St-Cyr Tribble D,Desrosiers J. Meaning of quality of life for older adults: Importance of human functioning components. Archives of gerontology and geriatrics. 2009; 49(2):91-100.

Bowling A, Banister D, Sutton S, Evans O, Windsor J. A multidimensional model of the quality of life in older age. Aging Mental Health. 2002; 6(4):355-71.

Khosrozadeh H, Alavi NM, Gilasi H, Izadi M. Oral health-related quality of life in older people in Kashan/Iran 2015.Nurs Midwifery Stud 2017;6:182-8.

SHARON GIL.Risk Factors for Traumatic Exposure and Posttraumatic Stress Symptoms. Journal of Loss and Trauma, 20:245-252, 2015

Weathers,FW,Litz,B.T.HermanD.S,Huska,J.A\&Keane.T.The PTSD Cheklist (PCL). Reliability. Validity \& diagnostic utility. Presented at the 9 Th Annual Meeting of the Inernational Society for Traumatic Seress Hissre Studies. October 1993. Sam Antunio, TX.

Üstün TB, Kostanjsek N, Chatterji S, Rehm J. Measuring health and disability: manual for WHO disability assessment schedule (WHODAS 2.0). Switzerland: WHO Library Cataloguing-in-Publication Data; 2010: 4-20.

Bredemeier J, Peretti Wagner G, Agranonik M, Spalding Perez T, Fleck PM. The world health organization quality of life instrument for people with intellectual and physical 
disabilities (WHOQOLDis): Evidence of validity of the Brazilian version. BMC Public Health. 2014; 14(1):538-58.

H.R. Baghaee, A.K. Kaviani, M. Mirsalim, G.B. Gharehpetian, "Harmonic optimization in single DC source multi-level inverters using RBF neural networks", in Proc. 3rd Power Elect. \& Drive Systems Technology (PEDSTC), Sharif Univ. of Techn., Tehran, Iran, Feb. 2012: 403 - 409.

H. Demuth, M. Beale, Neural Network Toolbox for use with MATLAB, The MathWorks, Natick, MA (2015).

S. Chen, C.F.N. Cowan, P.M. Grant, "Orthogonal least squares learning algorithm for radial basis function networks," IEEE Trans. on Neural Net., vol. 2, no. 2, pp. 302-309, Mar. 1991.

J.A. Leonard, "Radial basis function networks for classifying process faults", IEEE Trans. on Control Systems.April 1991:11(3): $31-38$.

I. Tarassenko, S. Roberts, "Supervised and unsupervised learning in radial basis function classifiers”, IET Proc. Vision, Image and Signal Processing.August 1994 :141(4): 210 216.

Pagulayan KF1, Temkin NR, Machamer J, Dikmen SS.A longitudinal study of health-related quality of life after traumatic brain injury. Arch Phys Med Rehabil. 2006 May;87(5):6118.

Truelle JL1, Fayol P, Montreuil M, Chevignard M. Community integration after severe traumatic brain injury in adults. Curr Opin Neurol. 2010 Dec;23(6):688-94. 
Xiao Zhou, Xinchun Wu, and Yuanyuan An.Understanding the Relationship between Trauma Exposure and Depression among Adolescents after Earthquake: The Roles of Fear and Resilience. Front Psychol. 2016; 7: 2044.

Gunning A, van Heijl M, van Wessem K, Leenen L. The association of patient and trauma characteristics with the health-related quality of life in a Dutch trauma population. Scand J Trauma Resusc Emerg Med. 2017 Apr 14;25(1):41. 


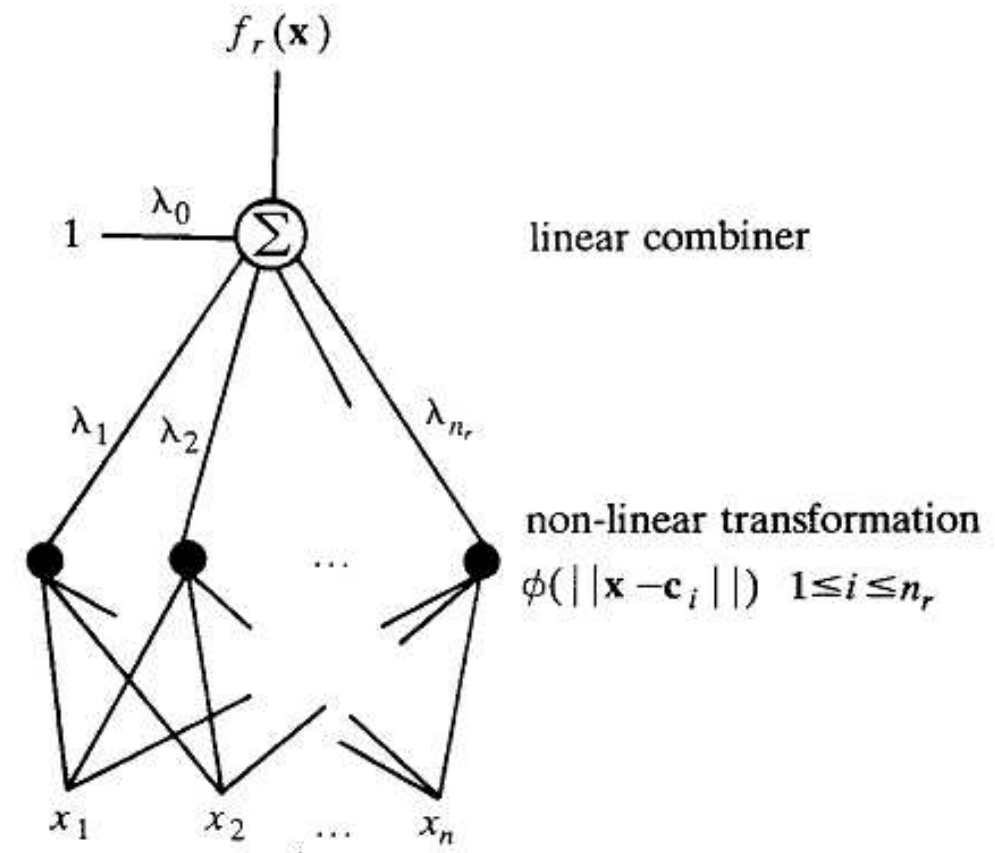

Fig. 1 Schematic of RBFNN 0 "(c) IEEE from 0". 


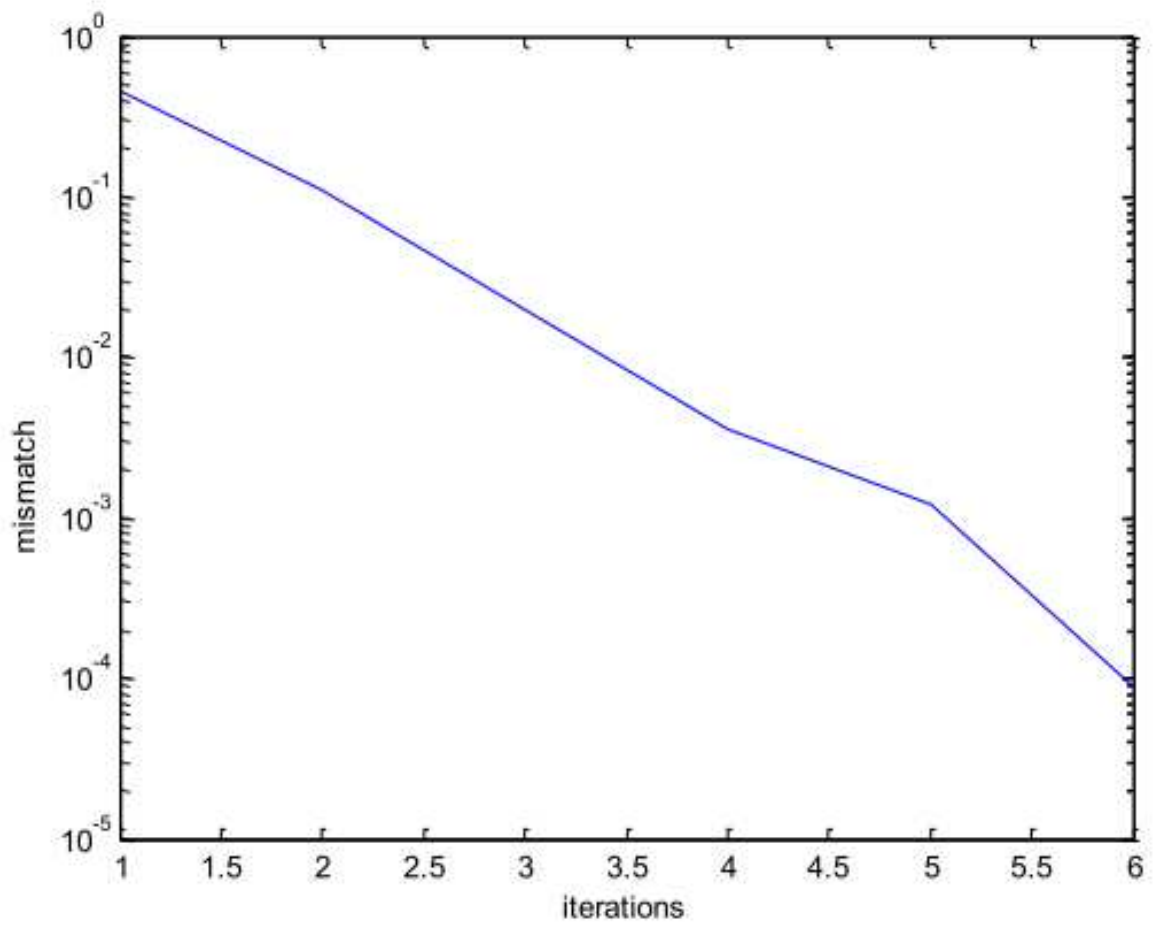

Fig. 2. Variations of mismatch versus iterations for the RBFNN predictor. 
Table 1- Compare mental health and quality of life in people with or without trauma

\begin{tabular}{|c|c|c|c|c|c|c|c|}
\hline & \multicolumn{2}{|c|}{ Trauma during past year ${ }^{*}$} & \multirow[t]{2}{*}{ OR } & \multicolumn{2}{|c|}{ CI $95 \%$} & \multirow{2}{*}{$P$-value } \\
\hline & & No & Yes & & Lower & Upper & \\
\hline \multirow{4}{*}{$\begin{array}{c}4 \text { section } \\
\text { of } \\
\text { Mental } \\
\text { Health }\end{array}$} & $\begin{array}{c}\text { Physical } \\
\text { Symptoms }\end{array}$ & $1844(51.1 \%)$ & $147(53.6 \%)$ & .904 & .707 & 1.157 & 0.42 \\
\hline & $\begin{array}{c}\text { Anxiety And } \\
\text { Insomnia }\end{array}$ & $1292(35.8 \%)$ & $83(30.3 \%)$ & 1.285 & .984 & 1.677 & 0.06 \\
\hline & $\begin{array}{c}\text { Inadequate Social } \\
\text { Action }\end{array}$ & $702(19.5 \%)$ & $38(13.9 \%)$ & 1.501 & 1.055 & 2.136 & 0.023 \\
\hline & Depression & $2622(72.7 \%)$ & $209(76.3 \%)$ & .829 & .621 & 1.105 & 0.2 \\
\hline \multicolumn{2}{|c|}{ Mental Health } & $1395(38.7 \%)$ & $92(33.6 \%)$ & 1.248 & .963 & 1.618 & 0.09 \\
\hline \multirow{3}{*}{$\begin{array}{c}\text { Quality Of } \\
\text { Life }\end{array}$} & Bad & $251(7.0 \%)$ & $45(16.4 \%)$ & 2.627 & 1.861 & 3.706 & 0.00 \\
\hline & Moderate & $1374(38.1 \%)$ & $134(48.9 \%)$ & 1.555 & 1.215 & 1.989 & 0.00 \\
\hline & Good & $1981(54.9 \%)$ & $95(34.7 \%)$ & .435 & .337 & .563 & 0.00 \\
\hline
\end{tabular}

${ }^{\mathrm{a}}$ Data are presented as No. (\%).

b Abbreviations: CI, Confidence Interval; OR, Odds Ratio. 


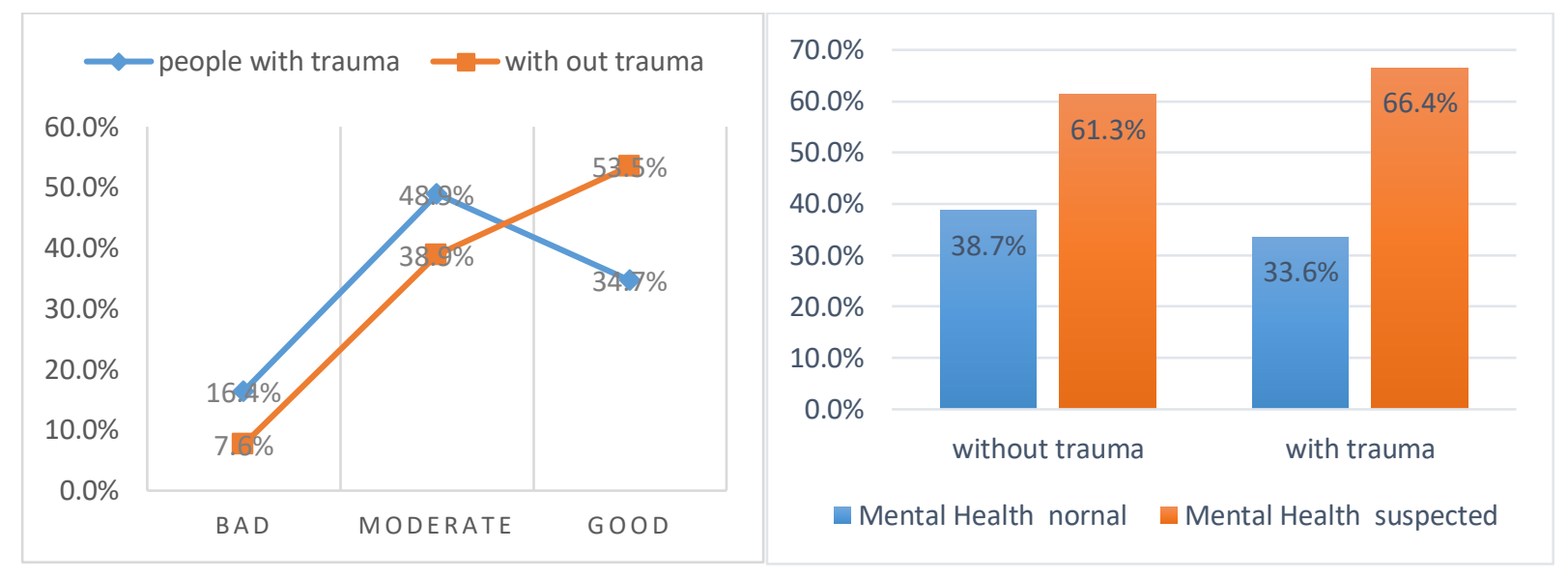

Fig. 3 Quality of life in people with or

Fig. 4 Mental health in people with or without trauma. without trauma. 
Table 2-Demographic characteristics based on Mental Health and quality of life status in people with trauma

\begin{tabular}{|c|c|c|c|c|c|c|c|c|}
\hline & \multicolumn{2}{|c|}{ Mental Health ${ }^{a}$} & \multirow[b]{2}{*}{$\begin{array}{c}P- \\
\text { value }\end{array}$} & \multicolumn{3}{|c|}{ Quality of life } & \multirow[b]{2}{*}{$\begin{array}{c}P- \\
\text { value }\end{array}$} \\
\hline & & $\begin{array}{c}\text { Suspected of } \\
\text { mental } \\
\text { disorder }\end{array}$ & Normal & & $\mathrm{Bad}$ & Moderate & Good & \\
\hline \multirow{2}{*}{ Sex } & Male & $82(38.5 \%)$ & $131(61.5 \%)$ & \multirow{2}{*}{0.01} & $29(13.6 \%)$ & $101(47.4 \%)$ & $83(38.96 \%)$ & \multirow{2}{*}{0.06} \\
\hline & Female & $10(16.4 \%)$ & $51(83.6 \%)$ & & $16(26.2 \%)$ & $33(54 \%)$ & $12(19.6 \%)$ & \\
\hline \multirow{3}{*}{ Age } & $15-29$ & $34(40.9 \%)$ & $50(59 \%)$ & \multirow{3}{*}{0.00} & $5(5.9 \%)$ & $44(52 \%)$ & $35(41.6 \%)$ & \multirow{3}{*}{0.00} \\
\hline & $30-49$ & $37(32.1 \%)$ & $78(67.8 \%)$ & & $21(9.4 \%)$ & $44(41.89 \%)$ & $50(48.6 \% \%)$ & \\
\hline & $50->70$ & $21(28.3 \%)$ & $53(71.6 \%)$ & & $19(33.3 \%)$ & $46(41.02 \%)$ & $10(25.64 \%)$ & \\
\hline \multirow{4}{*}{ Education } & Illiterate & $5(38.4 \%)$ & $8(61.53 \%)$ & \multirow{4}{*}{0.05} & $5(38.46 \%)$ & $8(61.53 \%)$ & 0 & \multirow{4}{*}{0.00} \\
\hline & Primary & $28(29.1 \%)$ & $68(70.9 \%)$ & & $30(31.25 \%)$ & $44(45.8 \%)$ & $22(22.9 \%)$ & \\
\hline & Diploma & $34(33.3 \%)$ & $68(66.6 \%)$ & & $5(4.9 \%)$ & $62(60.7 \%)$ & $35(34.31 \%)$ & \\
\hline & Academic & $25(39.68 \%)$ & $38(60.31 \%)$ & & $5(7.9 \%)$ & $20(31.74 \%)$ & $38(60.31 \%)$ & \\
\hline \multirow{2}{*}{ Marriage } & Single & $29(42.64 \%)$ & $39(57.35 \%)$ & \multirow{2}{*}{0.05} & $5(7.3 \%)$ & $29(42.64 \%)$ & $34(50 \%)$ & \multirow{2}{*}{0.00} \\
\hline & Marriage & $63(30.58 \%)$ & $143(69.41 \%)$ & & $40(19.41 \%)$ & $105(50.9 \%)$ & $61(29.61 \%)$ & \\
\hline \multicolumn{2}{|c|}{ Insurance } & $65(31.86 \%)$ & $139(68.13 \%)$ & 0.06 & $33(16.17 \%)$ & $104(50.98 \%)$ & $67(32.84 \%)$ & 0.05 \\
\hline \multicolumn{2}{|c|}{ Illness } & $15(23.43 \%)$ & $49(76.56 \%)$ & 0.04 & $19(29.68 \%)$ & $41(64 \%)$ & $5(7.8 \%)$ & 0.00 \\
\hline
\end{tabular}

${ }^{\mathrm{a}}$ Data are presented as No. $(\%)$ 
Table 3- Injury characteristics based on mental health and quality of life status

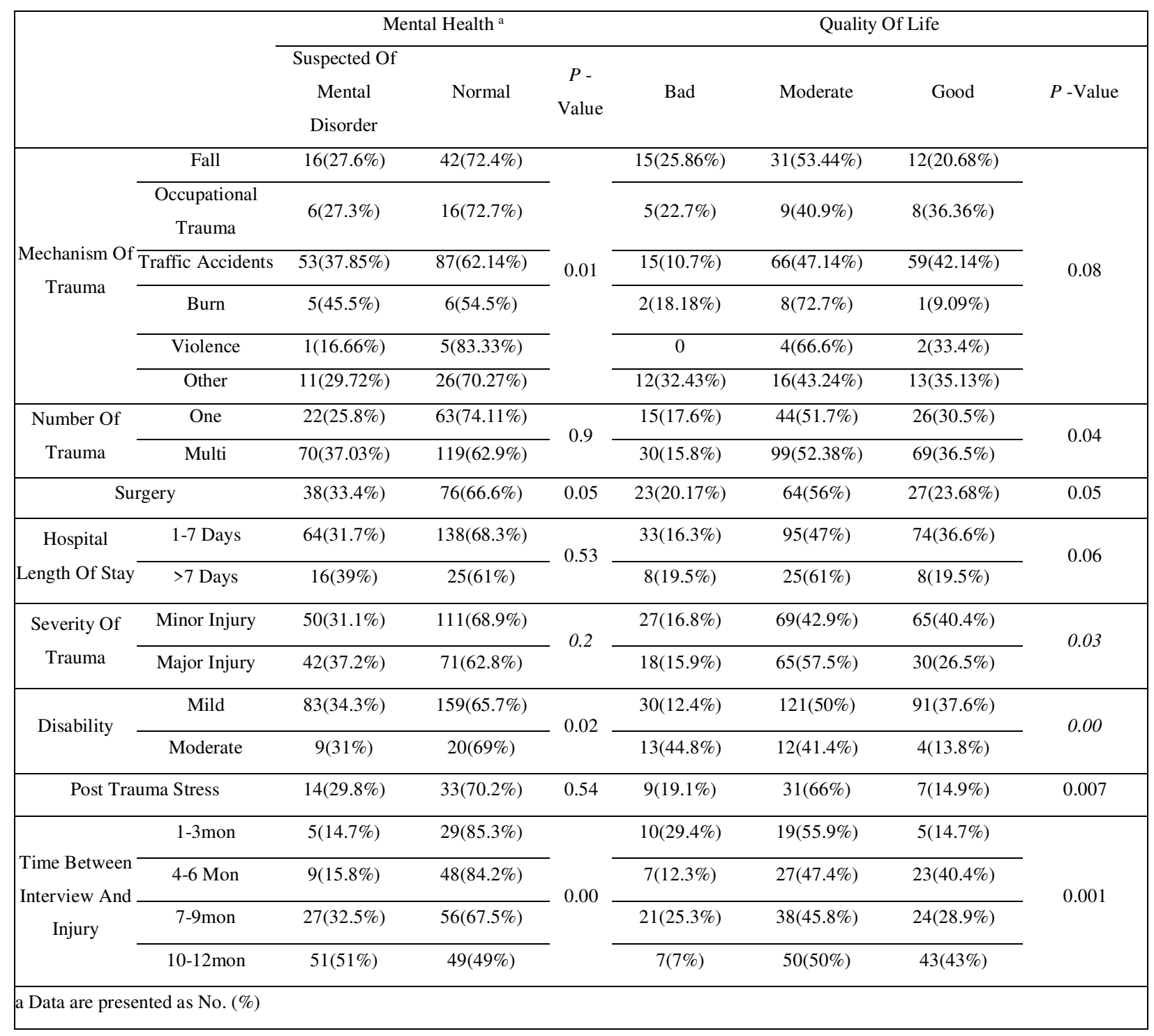


Table 4- Correlation between General Health and quality of life

\begin{tabular}{|lccc|}
\hline \multirow{2}{*}{ General Health } & \multicolumn{3}{c|}{ Quality of life } \\
\cline { 2 - 4 } & Bad & Moderate & Good \\
\hline Suspected Of Having Mental Disorder & $2.2 \%$ & $47.8 \%$ & $50 \%$ \\
\hline Normal & $423.6 \%$ & $49.5 \%$ & $26.9 \%$ \\
\hline Total & $16.4 \%$ & $48.9 \%$ & $34.7 \%$ \\
\hline
\end{tabular}

a Data are presented as (\%) 
Table 5-the values of Mental health and QOL predicted with RBFNN

\begin{tabular}{|c|c|c|c|}
\hline & & $\begin{array}{c}\text { Suspected } \\
\text { of Mental } \\
\text { Health }^{\mathrm{a}}\end{array}$ & $\begin{array}{c}\text { Moderate } \\
\text { and Good } \\
\text { Quality of } \\
\text { life }\end{array}$ \\
\hline Sex & Male & $40 \%$ & $47 \%$ \\
\hline & $>30$ years & $42 \%$ & $44 \%$ \\
\hline Age & $<30$ years & $58 \%$ & $43 \%$ \\
\hline & Primary & $32 \%$ & $45 \%$ \\
\hline Education & Academic & $39 \%$ & $55 \%$ \\
\hline Marriage & married) & $30 \%$ & $60 \%$ \\
\hline Insu & ince & $40 \%$ & $80 \%$ \\
\hline Illn & & $50 \%$ & $19 \%$ \\
\hline & Fall & $15 \%$ & $46 \%$ \\
\hline $\begin{array}{l}\text { Mechanism } \\
\text { Of Trauma }\end{array}$ & $\begin{array}{c}\text { Traffic } \\
\text { Accidents }\end{array}$ & $50 \%$ & $45 \%$ \\
\hline & Other & $30 \%$ & $37 \%$ \\
\hline Sur & & $33 \%$ & $20 \%$ \\
\hline D. & Mild & $30 \%$ & $50 \%$ \\
\hline & Moderate & $31 \%$ & $13 \%$ \\
\hline Post Trau & na Stress & $31 \%$ & $40 \%$ \\
\hline
\end{tabular}


Figures

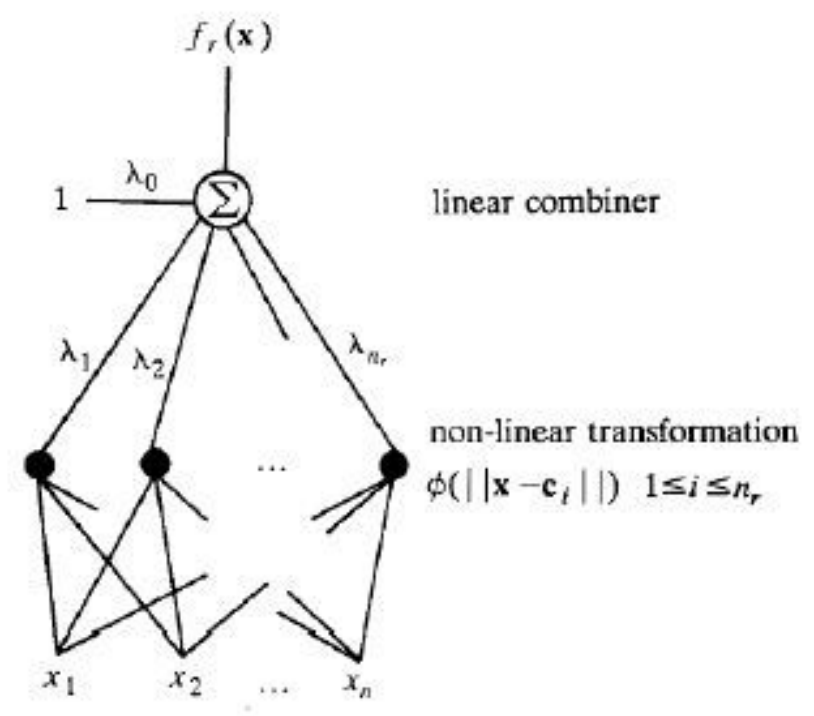

\section{Figure 1}

Schematic of RBFNN 0 "(c) IEEE from 0"

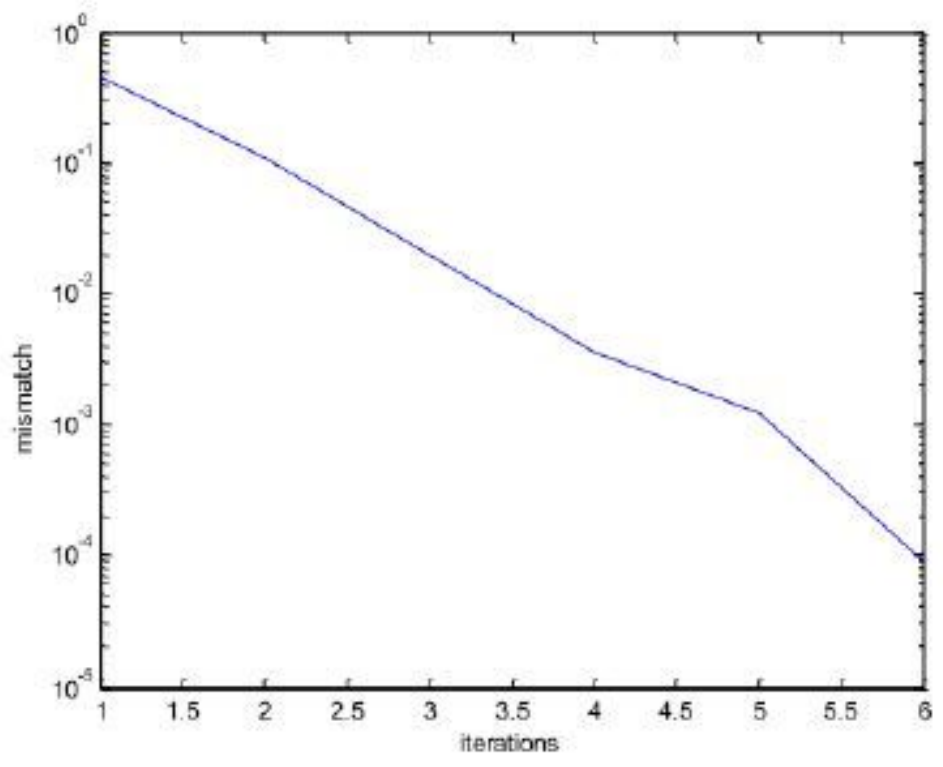

Figure 2

Variations of mismatch versus iterations for the RBFNN predictor 


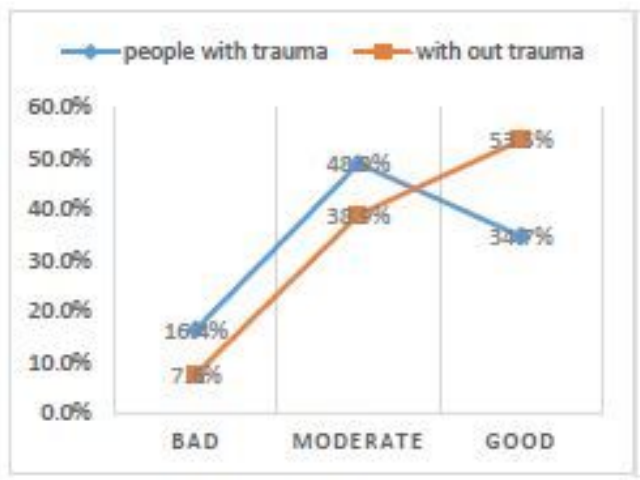

\section{Figure 3}

Quality of life in people with or without trauma

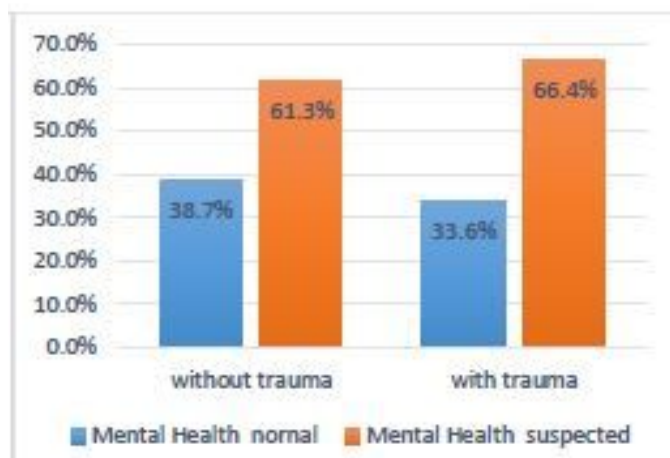

Figure 4

Mental health in people with or without trauma 